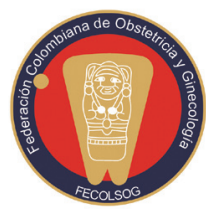

\title{
MECANISMOS DE MODULACIÓN DE LA RESPUESTA INMUNE POR CHLAMYDIA TRACHOMATIS ASOCIADOS A INFERTILIDAD
}

\section{Immune response modulation mechanisms induced by Chlamydia trachomatis associated with infertility}

Tatiana Marcela López-Castro, $M D^{I}$; Ever Leonardo Rojas-Díaz'; Fabio Noriel Rojas-Rojas, $M D^{3}$; Ivonne J. Díaz-Yamal, $M D^{4}$; Jairo Muñoz-Cerón, $M D^{5}$

Recibido: abril 12/12 - Aceptado: diciembre 13/12

\section{RESUMEN}

Introducción: la infertilidad, problema clínico y social que afecta del 13 al 15\% de las parejas en el mundo, es causada, entre otros, por la enfermedad pélvica inflamatoria ocasionada por varios agentes infecciosos entre los cuales se destaca la Chlamydia trachomatis. Este agente infeccioso posee mecanismos moleculares con los cuales modula la respuesta inmune del huésped y produce cambios en la célula infectada para permitir su supervivencia, ocasionando que la respuesta del sistema inmunológico se establezca en forma crónica, con la consecuente inflamación permanente y con ello secuelas como cicatrices y obstrucción de la trompa de Falopio. El objetivo de esta revisión es ofrecer una actualización de conocimiento en inmunobiología de la infección por Chlamydia trachomatis y su relación con la infertilidad.

Fellowship en salud reproductiva y humana, Fundación Universitaria Sanitas. Bogotá, Colombia. tamala2407@gmail.com

Estudiante X semestre, Facultad de Medicina, Fundación Universitaria Sanitas. Bogotá, Colombia.

Residente de Ginecología y Obstetricia I año, Fundación Universitaria Sanitas. Bogotá, Colombia.

Coordinadora Fellow en Salud Reproductiva y Humana, Fundación Universitaria Sanitas. Bogotá, Colombia.

Inmunólogo y microbiólogo. Docente, Fundación Universitaria Sanitas. Bogotá, Colombia.
Materiales y métodos: se realizó una revisión de la literatura en diferentes bases de datos: PubMed/ Medline, Science Direct, Ovid, desde enero del año 1995 a enero del 2012, incluyendo artículos de revisión y estudios clínicos.

Resultados: en la actualidad se sostiene que la inmunomodulación que caracteriza la infección por Chlamydia trachomatis, los mediadores inflamatorios implicados en la respuesta inmune, y la posible aunque poco estudiada susceptibilidad genética del huésped, se relacionan estrechamente con la génesis de la infertilidad por factor tubárico.

Conclusión: la infertilidad causada por Chlamydia trachomatis tiene su origen en la respuesta inmunológica del huésped y en la modulación por parte de este agente infeccioso, lo que lleva a inflamación crónica, cicatrización y obstrucción de la trompa de Falopio.

Palabras clave: Chlamydia trachomatis, infertilidad, inmunidad innata, inmunidad adquirida, infección.

\section{ABSTRACT \\ Introduction: Infertility is a clinical and social problem affecting $13 \%$ to $15 \%$ of couples around the world. One of its causes is the pelvic inflammatory disease, induced by several infectious agents, Chlamydia trachomatis standing out amongst}


them. This infectious agent has molecular mechanisms modulating the host immune response and producing changes in the infected cell to allow it to survive, casing a chronic immunological system response, with consequent permanent inflammation and sequelae such as cicatrices and obstruction of the fallopian tubes. This review was aimed at updating knowledge regarding the immune-biology of infection caused by Chlamydia trachomatis and its relationship with infertility.

Materials and methods: A literature review was made using PubMed/MEDLINE, Science Direct and Ovid databases from January 1995 to January 2012, including review articles and clinical studies.

Results: It is currently held that immunomodulation characterizing infection caused by Chlamydia trachomatis, the inflammatory mediators implicated in the immune response and possible (though little studied) host genetic susceptibility are closely related to the genesis of tubal infertility.

Conclusion: Infertility caused by Chlamydia trachomatis has its origin in a host's immunological response and this infections agent's modulation leading to chronic inflammation, healing and obstruction of the fallopian tubes.

Key words: Chlamydia trachomatis, infertility, innate immunity, acquired immunity, infection.

\section{INTRODUCCIÓN}

Chlamydia trachomatis (CT) es una bacteria que pertenece al género Chlamydia y a la familia Chlamydiaceae. Es un microorganismo intracelular obligado, debido a que carece de mecanismos para producir adenosin trifosfato (ATP). Dentro de sus características generales podemos mencionar que posee una morfología cocoide, es inmóvil y de tamaño aproximado de $0,4 \mu \mathrm{m}$, cuenta con una pared celular provista de membrana interna y externa la cual es rica en lipopolisacáridos. Al parecer carece de ácido nacetilmurámico componente del peptidoglicano, comportándose como una bacteria gram negativa débil (1-4). Se han descrito alrededor de 20 serotipos distintos, de los cuales los serotipos D, F, G,
H, I, J, K son catalogados como los responsables de infecciones sexualmente transmitidas e infección en neonatos (5).

Chlamydia trachomatis tiene un ciclo de desarrollo bifásico en donde se observan formas infecciosas, inactivas metabólicamente (cuerpos elementales, $\mathrm{CE})$, y formas no infecciosas, activas metabólicamente (cuerpos reticulados, CR). Los CE cuentan con proteínas de membrana con fuertes enlaces disulfuro cruzados, que le proporcionan gran afinidad por proteoglicanos de las células epiteliales del huésped en las que penetra rápidamente mediante adhesinas, receptores y otros mecanismos $(1,5)$. Después de entrar a la célula huésped las bacterias modifican el tráfico vesicular mediado por Rab-14 (Ras-related protein-14), asegurando su replicación dentro de una única vacuola, no degradativa, ni ácida, llamada inclusión, en la zona perigolgi (6-8). Si la membrana externa del CE está intacta se inhibe la fusión de los lisosomas celulares evitando la destrucción intracelular; cuando esta membrana se lesiona o se inactivan las bacterias mediante calor o recubrimiento por anticuerpos, se produce la fusión del fagosoma-lisosoma con su posterior destrucción. La reorganización de proteínas de membrana da lugar a los CR, que adquieren mayor actividad metabólica dividiéndose mediante fisión binaria restableciéndose nuevamente en CE, llevando a lisis celular y su posterior liberación, lo que permite la infección a nuevas células. Esta capacidad de CT para cambiar de su forma inactiva hasta su forma infectante replicativa hace que sea más difícil su eliminación (9).

La infertilidad, definida como la incapacidad de la pareja para concebir luego de un año de relaciones sexuales sin protección (10), continúa siendo un problema clínico y social que afecta del 13 al 15\% de las parejas en el mundo (11), las causas más comunes son: el factor masculino dado por anormalidades espermáticas, entre otras; el factor femenino como disfunción ovulatoria y patología tubárica; factores masculinos y femeninos combinados, e infertilidad inexplicada $(12,13)$. 
La enfermedad tubárica corresponde al 25-35\% de las causas de infertilidad femenina, pudiendo comprometer a la trompa en toda su extensión. La principal causa del compromiso tubárico es la enfermedad pélvica inflamatoria (EPI) $(14,15)$ ocasionada por Chlamydia trachomatis (CT) en un 40\% de los casos según estadísticas de los Estados Unidos (16), y con 90 millones de casos nuevos al año en el mundo (17). La incidencia de lesión tubárica después del primer episodio de infección por CT es aproximadamente del 12\%, incrementando desde un 23-54\% en los episodios posteriores (18, 19). Aproximadamente $75 \%$ de las mujeres y $50 \%$ de los hombres infectados son asintomáticos, lo cual hace que su diagnóstico y manejo tardío traiga consecuencias a futuro $(20,21)$. Un solo episodio de salpingitis por CT se autolimita, pero episodios posteriores pueden ocasionar cicatrización en las trompas incrementando el riesgo de infertilidad (14), esto significa que una enfermedad pélvica inflamatoria (EPI) sintomática no es prerrequisito para una lesión tubárica subsecuente, sugiriendo que la repuesta inmune innata y adaptativa frente a los antígenos inmunodominantes de CT juega un papel importante en la patogénesis de la infertilidad (22-26). Además, la infección por CT se asocia, como factor de riesgo independiente, con el desarrollo de neoplasias intraepiteliales del cérvix (27). Por lo anterior es importante que los profesionales de la salud y el personal en formación conozcan y tengan claridad sobre los mecanismos que CT utiliza para generar lesión tisular y vulnerar su erradicación por parte del sistema inmune, lo cual lleva a generar cuestionamiento sobre el papel actual del diagnóstico y tratamiento de este agente infeccioso. Por tanto, el objetivo de esta revisión es ofrecer una actualización de conocimiento en inmunobiología de la infección por Chlamydia trachomatis y su relación con infertilidad.

\section{MATERIALES Y MÉTODOS}

Se realizó una búsqueda en PubMed/Medline, Science Direct y Ovid empleando los siguientes términos MeSH: "chlamydia trachomatis", "infertility", "allergy and immunology", "innate immunity", "adaptive immunity", "infection" y "vaccines". Se eligieron artículos de revisión y estudios clínicos desde enero del año 1995 a enero del 2012 que trataron sobre la relación de Chlamydia trachomatis e infertilidad y la respuesta inmunológica.

\section{RESULTADOS}

Se encontraron 160 referencias de las cuales se eligieron 66 que cumplían con el objetivo planteado para esta revisión. Tras una evaluación y recopilación de la literatura se presentan a continuación los resultados encontrados.

\section{Respuesta inmune frente a Chlamydia trachomatis}

Chlamydia trachomatis puede permanecer en el tejido inflamado del tracto genital superior de las pacientes infectadas, ya que se ha reportado una tasa de depuración de tan solo el 44,7\% en pacientes asintomáticas y sin tratamiento, en un seguimiento hasta por un año $(27,28)$.

\section{Respuesta inmune innata}

Según lo publicado en estudios recientes, una de las principales puertas de acceso de CT al tracto genital es mediante su adherencia a espermatozoides, convirtiéndolos en agentes activos de la transmisión de la infección. La infección inicia en las células epiteliales del endocérvix, en donde los CE interaccionan con receptores tipo Toll (Toll-like receptor TLRs) (29, 30). Estudios in vitro con líneas celulares epiteliales inmortalizadas derivadas del tejido endocervical concluyen que la producción de mucina, factor protector durante la infección por CT y primera línea de defensa, es dependiente de múltiples factores como: receptores específicos para hormonas esteroideas, expresión de TLR-3 (Toll-like receptor 3), producción de péptidos antimicrobianos y citocinas proinflamatorias, lo que a su vez parece ser vulnerado durante la infección por CT o podría ser un factor, que al estar deficiente en el huésped, incrementaría 
la susceptibilidad a la infección (31). Durante la infección por CT existe activación de TLR-2 y TLR-4, los cuales a su vez inducen activación y translocación del factor de transcripción NF- $\kappa \mathrm{B}$ (nuclear factor kappalight-chain-enhancer of activated $\mathrm{B}$ cells) al núcleo, activando secuencias génicas que conducen a la síntesis de Interleucina 8 (L-8), citocina proinflamatoria que participa en la depuración del agente infeccioso (32-34). Chlamydia trachomatis prefiere la activación de TLR-2, lo cual se ha asociado con producción de óxido nítrico, especie reactiva del metabolismo del nitrógeno con acción bactericida $(35,36)$.

A diferencia de otros modelos infecciosos, la presencia de plásmidos en CT se encuentra asociada a menor patogenicidad debido a que estos pueden regular de forma negativa genes cromosómicos, como el de la sintetasa de glucógeno, limitando la glucosa en la célula bacteriana (37) y, además, porque participan en la activación de TLR-2 y la consecuente producción de citocinas, por tanto, la expresión de plásmidos es inversamente proporcional a la patología en el aparato genital alto (38); sin embargo, la deficiencia de plásmidos es muy infrecuente y solo se ha observado en 3 cepas de CT (39-41). Polimorfismos genéticos de TLR-1 y TLR-4 se han asociado con incremento en la susceptibilidad a la infección en población afroamericana (42).

Una vez que los cuerpos elementales ingresan a las células epiteliales y a los macrófagos locales, se induce la producción de citocinas -IL-1,FNT- $\alpha$ (tumor necrosis factor $\alpha$ ), GRO- $\alpha$ (growth-related oncogene $\alpha$ )- que promueven la expresión de moléculas de adhesión -ICAM-1 (InterCellular Adhesion Molecule-1), VCAM-1 (Vascular Cell Adhesion Molecule-1), MadCAM-1 (Human mucosal addressin cell adhesion molecule-1) - atrayendo células fagocíticas como los polimorfonucleares, que a su vez se encargan de producir especies reactivas del metabolismo del oxígeno (EROS) y metaloproteasas que contribuyen a la lesión y el remodelamiento tisular $(43,44)$. En modelos experimentales con células del epitelio túbarico humano, infectadas con CT y expuestas a azitromicina, se observa que aun después de la destrucción bacteriana persisten ciertas proteínas de CT que promueven la actividad proinflamatoria de los neutrófilos y células asesinas naturales (Natural Killer) (45).

Posterior al establecimiento de la respuesta inmune innata se genera la respuesta inmune adaptativa en donde el protagonista es el linfocito $\mathrm{T}$.

\section{Respuesta inmune adaptativa}

Como en varios modelos de infección, es importante citar que durante la primoinfección el redireccionamiento de la repuesta de linfocitos $T$ ayudadores 1 o 2 (limphocyte T helper Th-1, Th-2) es importante en la patogénesis de la infección por CT (43). Se ha demostrado que la respuesta Th1, caracterizada por la producción de interferon e IFN- $\gamma$ está asociada con depuración y control de la reinfección. En modelos murinos infectados por chlamydia se ha observado diferente respuesta inmunológica en el tracto genital alto (TGA) frente al tracto genital bajo (TGB). En el TGA se observa una mayor expresión del RNAmensajero ( $m R N A)$ del factor de transcripción T-bet (transcription factor T-bet), de INF- $\gamma$ y de Fox-P3 (forkhead box P3), lo que al parecer sugiere un ambiente proinflamatorio que cuenta con la presencia de linfocitos T reguladores. Mientras que en TGB existe una mayor expresión del $m R N A$ del factor de transcripción GATA-3 (Trans-acting T-cell-specific transcription factor 3), e IL-10, la cual es producida por células dendríticas CD-11c ${ }^{+} \mathrm{CD}-11 \mathrm{~b}^{+}$, dominando una respuesta Th2, sin ser la ideal frente a un agente infeccioso intracelular (43-47).

Como se mencionó, el perfil de citocinas que se produce en el TGA es de predominio proinflamatorio y, además del INF- $\gamma$, se producen otras citocinas como la IL-1, IL-8 y IL-12 que llevan a la consecuente lesión tisular durante la infección primaria, buscando la depuración del agente infeccioso (43, 44). A su vez, estas citocinas en conjunto con GMcsf (Granulocyte-macrophage colony-stimulating factor) y la IL-6, hacen que perdure la respuesta hasta por 24 horas después de la primoinfección, en comparación con otras bacterias cuya duración es menor. 
Posterior a la primoinfección existe una perpetuación en la producción de citocinas proinflamatorias mediada por IL-1 alfa, la cual se convierte en un amplificador de la respuesta mediada por citocinas, en especial su producción por parte de células que no se encuentran infectadas (45).

Chlamydia trachomatis produce la proteína de choque térmico 60 (del inglés $C$. trachomatis Heat shock proteins 60, CHSP-60) la cual genera una reacción de hipersensibilidad retardada. Se ha demostrado gran similitud entre una proteína de choque térmico producida por las células epiteliales y los macrófagos con la CHSP-60 de CT, lo cual se relaciona con mimetismo molecular y reafirma la teoría de autoinmunidad durante la infección persistente por CT. Además, es bien demostrado que CT permanece en un estado de latencia con inmunogenicidad baja pero persistente (43-47), debido a que en la célula infectada existe una depleción en la concentración de triptófano -aminoácido vital para CT-dada por la actividad de IDO (indoleamine 2,3-dioxygenase) inducida por el INF-gamma, que a su vez es producido por los LT CD4+. Sin embargo, CT cuenta con la capacidad de reactivar la síntesis de triptófano a través del metabolismo INDOL (48).

Durante la infección persistente hay una mayor expresión de las proteínas de choque térmico 10 (CHSP-10) y CHSP60 de CT, las cuales inducen una gran producción de anticuerpos, lo que no ocurre durante la infección aguda en donde predominan anticuerpos contra MOMP proteínas de pared de CT (Major Outter Membrane Protein) (49-51). Por otra parte, la gran expresión de estas proteínas genera una gran proliferación de LT CD4+ Th1 hiperproductores de IL-2 e IFN- $\gamma$, situación asociada con la magnitud del daño tisular persistente, el cual se encuentra asociado con cicatrización y posterior oclusión de la trompa $(8,52)$.

Además, se ha postulado que la primoinfección es responsable en parte de la magnitud de la lesión tisular en infecciones posteriores, debido a que se genera, a partir del primer contacto con CT, una gran respuesta de linfocitos $\mathrm{T}$ de memoria los cuales, incluso durante contactos posteriores con CT, son más rápidos y efectivos que células como los neutrófilos; de hecho, el número de infecciones se asocia con una mayor respuesta de memoria de linfocitos $\mathrm{T}$ específicos $(53,54)$.

Con respecto a los LT CD8+ durante la infección persistente, en modelos murinos predominan en comparación con LTCD4+, no obstante, su papel en humanos es aún desconocido. Finalmente, los LTh17 podrían estar implicados en el reclutamiento de neutrófilos en la mucosa tubárica durante la infección persistente, entendiendo que estas células producen interleucina-17, responsable de este reclutamiento. Sin embargo, faltan estudios que puedan concluir aspectos cruciales sobre la actividad Th17 en esta patología $(55,56)$.

\section{Respuesta inmune humoral durante la infección por $\mathrm{CT}$}

Con relación a la respuesta humoral frente a CT, se ha encontrado que las mujeres con infertilidad y patología tubárica tienen mayor producción de anticuerpos del isotipo Inmunoglobulina $\mathrm{G}(\lg G)$ frente a CT, frente a aquellas mujeres con infertilidad, en ausencia de patología tubárica (52). Sin embargo, no existe claridad acerca de si este tipo de respuesta humoral es protectora o contrariamente asociada a mayor susceptibilidad. En la actualidad hay varias técnicas para la detección de anticuerpos antichlamydia y su relación con infertilidad, ya que se encuentran elevados en un $70 \%$ de mujeres con oclusión tubárica (57), siendo el más común la detección de IgG anti-CHSP60 (58).

\section{Modulación de la respuesta inmune del huésped por Chlamydia trachomatis}

Chlamydia trachomatis utiliza mecanismos para evadir la respuesta inmune y producir cambios celulares que permitan su supervivencia para perpetuar la inflamación. Se ha encontrado que uno de los mecanismos que usa CT para evadir la respuesta inmune de reconocimiento y destrucción por los linfocitos T citotóxicos es la regulación negativa de la expresión 
del complejo mayor de histocompatibilidad mayor $(\mathrm{CMH})$ clase I, alterando la presentación antigénica; sin embargo, esta disminución lleva a una activación de las natural killer (NK), en donde se encuentran implicados polimorfismos de una molécula no clásica del complejo mayor de histocompatibilidad llamada MICA (MHC class I chain-related gene A), la cual es un ligando activador de las NK, esto se encuentra asociado con lesión tisular (59).

Otro mecanismo de CT es contrarrestar la apoptosis de la célula huésped bloqueando la liberación del citocromo $\mathrm{C}$ desde la mitocondria hacia el citosol, e inhibiendo la activación de la caspasa 3 y su señal proapoptótica, con el fin de completar su ciclo reproductivo intracelular $(28,60,61)$.

Chlamydia trachomatis posee un set de proteínas que actúan en forma primaria como metiltransferasas de histonas. Durante la infección, estas proteínas se translocan al núcleo de la célula huésped y se asocian con la cromatina; su actividad enzimática permite unir grupos metilo a residuos de lisina de las histonas produciendo modificaciones en la acetilación, fosforilación y metilación, contribuyendo a la activación o supresión de genes de forma directa o indirecta, por un cambio en la estructura de la cromatina, esto permite a las bacterias resistir a los mecanismos inmunológicos de huésped (62).

Chlamydia trachomatis modula de forma negativa la respuesta de NF- $\kappa \mathrm{B}$ mediante su interacción con la proteína inhibidora $\mathrm{B}$, favoreciendo su sobrevida dentro del huésped ya que evita la expresión de genes que codifican para citocinas proinflamatorias, quimiocinas, moléculas de adhesión y reguladores de apoptosis, las cuales tienen como función principal reclutar macrófagos y polimorfonucleares, con el fin de erradicar la infección (63).

Por otra parte, existen estudios en donde se demuestra que el difosforil-lípido A, componente de la pared de CT, antagoniza el TLR-4 disminuyendo de forma parcial la expresión del receptor de INF- $\gamma$, citocina proinflamatoria que participa en el control de la infección por CT (64). Finalmente, CT incrementa la longevidad de los polimorfonucleares, la producción de citocinas y metaloproteinasa-9 de matriz (MMP9) vía dependiente de TLR-2, además de encontrarse una mayor actividad de la oxidasa fagocitaria, con gran producción de especies reactivas de oxígeno (EROS), lo cual se encuentra asociado con lesión tisular $(65,66)$ (tabla 1).

\section{Tabla 1.}

\section{Mecanismos de modulación de la respuesta inmune del huésped por Chlamydia trachomatis}

\section{Relacionadas con su supervivencia:}

1. Regulación negativa de la expresión del complejo mayor de histocompatibilidad (CMH) clase I.

2. Inhibición de la apoptosis de la célula del huésped.

3. Metilación de ADN, contribuyendo a la activación o supresión de genes del huésped.

4. Inhibición de NFKB mediante su interacción con la proteína inhibidora B.

5. Difosforil-lípido A antagoniza TLR-4.

\section{Relacionadas con mayor daño tisular:}

1. Modulación positiva de la actividad de las NK.

2. Incremento de la longevidad de los polimorfonucleares.

3. Incremento de la producción de citocinas proinflamatorias.

4. Incremento de la producción de metaloproteinasa-9 de matriz (MMP9) vía dependiente de TLR-2.

5. Incremento de la actividad de la oxidasa fagocitaria, con gran producción de especies reactivas del metabolismo del oxígeno.

6. Homología de HSP60 de CT y humana. 


\section{CONCLUSIONES}

La infertilidad como consecuencia de la infección por Chlamydia trachomatis tiene su origen en la intensidad de la respuesta inmunológica del huésped, asociado a mecanismos utilizados por el agente infeccioso para sobrevivir dentro de la célula epitelial y mantener en forma crónica los procesos de inflamación que llevan a la cicatrización y obstrucción de la trompa de Falopio.

La depuración total del agente infeccioso es un factor que podría estudiarse, ya que está directamente asociado con la patología resultante, por tanto, los protocolos de tratamiento antibiótico deberían ser reevaluados, así como los métodos diagnósticos serológicos empleados.

No existen estudios con información clara sobre el comportamiento en los títulos de anticuerpos, tiempo de seroconversión, y duración de la serorreactividad en mujeres con infección persistente por Chlamydia trachomatis, hecho que abre las puertas a los investigadores en este tema.

La complejidad de dichos mecanismos inmunes, tanto del huésped como de la bacteria, ha impedido la creación de una vacuna efectiva, la cual tendría más efectividad si se usara antes del primer contacto sexual, permitiendo prevenir la infección y con ello disminuir la incidencia de infertilidad por este factor.

\section{REFERENCIAS}

1. Abdel Rahman YM, Belland RJ. The chlamydial developmental cycle FEMS Microbiol Rev 2005;29:949-59.

2. Centers for Disease Control, Division of Sexually transmitted Diseases: STD treatment guidelines. MMWR 1995;34(4S).

3. Raulston JE. Chlamydial envelope components and pathogen - host cell interactions. Mol Microbiol 1995;15:607-16.

4. Nichols BA, Setzer PY, Pang F, Dawson CR. New view of the surface projections of Chlamydia trachomatis. J Bacteriol 1998;164:344-9.

5. Ghuysen JM, Goffin C. Lack of cell wall peptidoglycan versus penicillin sensitivity: new insights into the chlamydial anomaly. Antimicrob Agents Chemother 1999;43:2339-44.
6. Schachter J. Infection and disease epidemiology. En: Stephens RS. Editor. Chlamydia: intracellular biology, pathogenesis and immunity. Washington, DC: American Society of Microbiology; 1999. p. 139-69.

7. Capmany A, Leiva N, Damiani MT. Golgi-associated Rab14, a new regulator for Chlamydia trachomatis infection outcome. Commun Integr Biol 2011;4:590-3.

8. Linhares IM, Witkin SS. Immunopathogenic consequences of Chlamydia trachomatis $60 \mathrm{kDa}$ heat shock protein expression in the female reproductive tract. Cell Stress and Chaperones 2010;15:467-73.

9. Morré SA, van den Brule AJ, Rozendaal L, Boeke AJ, Voorhorst FJ, de Blok S, et al. The natural course of asymptomatic Chlamydia trachomatis infections: 45\% clearance and no development of clinical PID after one-year follow-up. Int J STD AIDS 2002;13:12-8.

10. Kamel RM. Management of the infertile couple: an evidence-based protocol. Reprod Biol Endocrinol 2010;8:21.

11. Irvine $S$. Guidelines in the treatment of male infertility. Int Cong Series 2004;1266:202-7.

12. Jose-Miller AB, Boyden JW, Frey KA. Infertility. Am Fam Physician 2007;75;849-56.

13. Wilkes S, Chinn DJ, Murdoch A, Rubin G. Epidemiology and management of infertility: a population-based study in UK primary care. Fam Pract 2009; 26:269-74.

14. Vásquez RA. Infertilidad de origen tubárico: diagnóstico y manejo. Rev Ces Med 2008;22:45-55.

15. Kodaman PH, Arici A, Seli E. Evidence-based diagnosis and management of tubal factor infertility. Curr Opin Obstet Gynecol 2004;16:221-9.

16. Oakley L, Doyle P, Maconochie N. Lifetime prevalence of infertility and infertility treatment in the UK: results from a population-based survey of reproduction. Hum Reprod 2008;23:447-50.

17. World Health Organization. Global Prevalence and Incidence of Selected Sexually Transmitted Diseases: Overviews and Estimates, Geneva: WHO; 1996.

18. Cohen CR, Brunham RC. Pathogenesis of chlamydia induced pelvic inflammatory disease. Sex Transm Infect 1999;75:21-4. 
19. Muzzi L, Sereni MI, Battista C, Zullo MA, Tambone V, Angioli R. Tubo-peritoneal factor of infertility: diagnosis and treatment. Clin Ter 2010;161:77-8.

20. Ruiz AI, Sánchez R, Ostos O, Ángel E, Bonilla H, Cifuentes C, et al. Estudio piloto de prevalencia de infección por chlamydia trachomatis detectada por PCR en mujeres con parto prematuro en el Instituto Materno Infantil de Bogotá. Rev Colomb Obstet Ginecol 2005;56:225-30.

21. Carey AJ, Beagley KW. Chlamydia trachomatis, a hidden epidemic: effects on the female reproduction and options for treatment. Am J Reprod Immunol 2010;63:576-86.

22. Brunham RC, Rekart ML. Considerations on Chlamydia trachomatis disease expression. FEMS Immunol Med Microbiol 2009;55:162-6.

23. Morrison SG, Morrison RP. In situ analysis of the evolution of the primary immune response in murine Chlamydia trachomatis genital tract infection. Infect Immun 2000;68:2870-9.

24. Kelly KA. Cellular immunity and Chlamydia genital infection: induction, recruitment, and effector mechanisms. Int Rev Immunol 2003;22:3-41.

25. Paavonen J, Weggert K. Chlamydia trachomatis: impact on reproduction human. Hum Reprod Update 1999;5:433-47.

26. Reddy B, Rastogi S, Das B, Salhan S, Verma S, Mittal A. Cytokine expression pattern in the genital tract of Chlamydia trachomatis positive infertile women implication for t-cell responses. Clin exp immunol 2004; 137:552-8.

27. Tavares BM, De Resende AD, Cunha FQ, Murta EF. Local profile of cytokines and nitric oxide in patients with bacterial vaginosis and cervical intraephitelial neoplasia. Eur J Obstet Gynecol Reprod Biol 2008;138:93-9.

28. Mei B, Luo Q, Du K, Huo Z, Wang F, Yu P. Association of mica gene polymorphisms with Chlamydia trachomatis infection and related tubal pathology in infertile women. Hum Reprod 2009;24:3090-5.

29. Carey AJ, Beagley KW. Chlamydia trachomatis, a hidden epidemic: effects on the female reproduction and options for treatment. Am J Reprod Immunol 2010;63:576-86.

30. Vigil P, Morales P, Tapia A, Riquelme R, Salgado AM. Chlamydia trachomatis infection in male partners of infertile couples: incidence and sperm function. Andrologia 2002;34:155-61.

31. Buckner LR, Schust DJ, Ding J, Nagamatsu T, Beatty W, Chang TL, et al. Innate immune mediator profiles and their regulation in a novel polarized immortalized epithelial cell model derived from human endocervix. J Reprod Immunol 2011;92:8-20.

32. Da Costa CU, Wantia N, Kirschning CJ, Busch DH, Rodriguez N, Wagner H, et al. Heat shock protein 60 from Chlamydia pneumoniae elicits an unusual set of inflammatory responses via Toll-like receptor 2 and 4 in vivo. Eur J Immunol 2004;34:2874-84.

33. Sasu S, LaVerda D, Qureshi N, Golenbock DT, Beasley D. Chlamydia pneumoniae and chlamydial heat shock protein 60 stimulate proliferation of human vascular smooth muscle cells via Toll-like receptor 4 and p44/ p42 mitogen-activated protein kinase activation. Circ Res 2001;89:244-50.

34. O'Connell CM, Ionova IA, Quayle AJ, Visintin A, Ingalls RR. Localization of TLR2 and MyD88 to Chlamydia trachomatis inclusions: evidence for signaling by intracellular TLR2 during infection with an obligate intracellular pathogen. J Biol Chem 2006;281:1652-9.

35. Agrawal T, Bhengraj AR, Vats V, Salhan S, Mittal A. Expression of TLR 2, TLR 4 and iNOS in cervical monocytes of Chlamydiatrachomatis-infected women and their role in host immune response. Am J Reprod Immunol 2011;66:534-43.

36. Prebeck S, Kirschning C, Dürr S, da Costa C, Donath B, Brand K, et al. Predominant Role of Toll-Like Receptor 2 Versus 4 in Chlamydia pneumoniaeInduced Activation of Dendritic Cells. J Immunol 2001;167;3316-23.

37. Matsumoto A, Izutsu H, Miyashita N, Ohuchi M. Plaque formation by and plaque cloning of Chlamydia trachomatis Biovar trachoma. J Clin Microbiol 1998;36:3013-9. 
38. O'Connell CM, AbdelRahman YM, Green E, Darville HK, Saira K, Smith B, et al. Toll-Like Receptor 2 Activation by Chlamydia trachomatis Is Plasmid Dependent, and Plasmid-Responsive Chromosomal Loci Are Coordinately Regulated in Response to Glucose Limitation by C. trachomatis but not by C. muridarum. Infect Immun 2011;79:1044-52.

39. Farencena AM, Comanducci M, Donati G, Ratti G, Cevenini R. Characterization of a new isolate of Chlamydia trachomatis which lacks the common plasmid and has properties of biovar trachoma. Infect Immun 1997;65:2965-9.

40. Peterson EM, Markoff BA, Schachter J, de la Maza LM. The 7.5-kb plasmid present in Chlamydia trachomatis is not essential for the growth of this microorganism. Plasmid 1990;23:144-8.

41. Stothard DR, Williams JA, van der Pol B, Jones RB. Identification of a Chlamydia trachomatis serovar E urogenital isolate which lacks the cryptic plasmid. Infect Immun 1998;66:6010-3.

42. Taylor BD, Darville T, Ferrell RE, Kammerer CM, Ness RB, Haggerty CL. Variants in toll-like receptor 1 and 4 genes are associated with Chlamydia trachomatis among women with pelvic inflammatory disease. J Infect Dis 2012;205:603-9.

43. Agrawal T, Vats V, Salhan S, Mittal A. The mucosal immune response to chlamydia trachomatis infection of the reproductive tract in women. J Reprod Immunol 2009;83:173-8.

44. Stephens RS. The cellular paradigm of chlamydial pathogenesis. Trends Microbiol 2003;11:45-9.

45. Rasmussen SJ, Eckmann L, Quayle AJ, Shen L, Zhang YX, Anderson DJ, et al. Secretion of proinflammatory cytokines by epithelial cells in response to Chlamydia infection suggests a central role for epithelial cells in chlamydial pathogenesis. J Clin Invest 1997;99:77-87.

46. Agrawal T, Vats V, Wallace PK, Singh A, Salhan S, Mittal A. Recruitment of myeloid and plasmacytoid dendritic cells in cervical mucosa during chlamydia trachomatis infection. Clin Microbiol Infect 2009;15:50-9.

47. Marks E, Tam MA, Lycke NY. The Female Lower Genital Tract Is a Privileged Compartment with IL-10
Producing Dendritic Cells and Poor Th1 Immunity following Chlamydia trachomatis Infection. PLoS Pathog 2010;6:e1001179.

48. Leonhardt RM, Lee SJ, Kavathas PB, Cresswell P. Severe tryptophan starvation blocks onset of conventional persistence and reduces reactivation of Chlamydia trachomatis. Infect Immun 2007;75:5105-17.

49. Agrawal T, Vats V, Salhan S, Mittal A. Mucosal and peripheral immune responses to chlamydial heat shock proteins in women infected with Chlamydia trachomatis. Clin Exp Immunol 2007;148:461-7.

50. Agrawal T, Vats V, Salhan S, Mittal A. Primary and secondary immune response of mucosal and peripheral lymphocytes during Chlamydia trachomatis infection. FEMS Immunol Med Microbiol 2007;49:280-7.

51. LaVerda D, Albanese LN, Ruther PE, Morrison SG, Morrison RP, Ault KA, et al. Seroreactivity to chlamydia trachomatis hsp10 correlates with severity of human genital tract disease. Infect Immun 2000;68:303-9.

52. Wyrick PB. Chlamydia trachomatis persistence in vitro: an overview. J Infect Dis 2010;15;201-16.

53. Kinnunen A, Paavonen J, Surcel HM. Heat shock protein 60 specific T-cell response in chlamydial infections. Scand J Immunol 2001;54:76-81.

54. Darville T, Hiltke TJ. Pathogenesis of genital tract disease due to Chlamydia trachomatis. J Infect Dis 2010;114-25.

55. Belland RJ, Scidmore MA, Crane DD, Hogan DM, Whitmire W, McClarty G, et al. Chlamydia trachomatis cytotoxicity associated with complete and partial cytotoxin genes. Proc Natl Acad Sci USA 2001;98:13984-9.

56. Crowley-Nowick PA, Ellenberg JH, Vermund SH, Douglas SD, Holland CA, Moscicki AB. Cytokine profile in genital tract secretions from female adolescents: impact of human immunodeficiency virus, human papillomavirus, and other sexually transmitted pathogens. J Infect Dis 2000; 181:939-45.

57. Rodgers AK, Wang J, Zhang Y, Holden A, Berryhill B, Budrys NM, et al. Association of tubal factor infertility with elevated antibodies to Chlamydia trachomatiscaseinolytic protease P. Am J Obstet Gynecol 2010;203:494.e7-494.e14. 
58. Stephens AJ, Aubuchon M, Schust DJ. Antichlamydial Antibodies, Human Fertility, and Pregnancy Wastage. Infect Dis Obstet Gynecol 2011; 2011:525182.

59. Fan T, Lu H, Hu H, Shi L, McClarty GA, Nance DM, et al. Inhibition of apoptosis in chlamydia-infected cells: blockade of mitochondrial cytochrome $\mathrm{C}$ release and caspase activation. J Exp Med 1998;187:487-96.

60. Fischer SF, Vier J, Kirschnek S, Klos A, Hess S, Ying $\mathrm{S}$, et al. Chlamydia inhibit host cell apoptosis by degradation of proapoptotic bh3-only proteins. J Exp Med 2004;200:905-16.

61. Rudel T, Kepp O, Kozjak-Pavlovic V. Interactions between bacterial pathogens and mitochondrial cell death pathways. Nat Rev Microbiol 2008;8: 693-705.

62. Pennini ME, Perrinet S, Dautry-Varsat A, Subtil A. Histone methylation by NUE A novel nuclear effector of the intracellular pathogen chlamydia trachomatis. Plos Pathog 2010;6:e1000995.
63. Rahman M, Mcfadden G. Modulation of NF-KB signalling by microbial pathogens. Nature Rev Microbiol 2011;9:291-306.

64. Hirschfeld M, Kirschning CJ, Schwandner R, Wesche $\mathrm{H}$, Weis JH, Wooten RM, et al. Inflammatory signaling by Borrelia burgdorferi lipoproteins is mediated by Toll-like receptor 2. J Immunol 1999;163:2382-6.

65. Frazer L, O’Connell C, Darville T. Chlamydialinduced TLR2 signaling leads to increased neutrophil production of proinflammatory molecules and delayed spontaneous apoptosis. J Immunol 2009;182:135-9.

66. Agrawal T, Gupta R, Dutta R, Srivastava P, Bhengraj AR, Salhan S, et al. Protective or pathogenic immune response to genital chlamydial infection in women-a possible role of cytokine secretion profile of cervical mucosal cells. Clin Immunol 2009;130:347-54. 